\title{
PEMODELAN PEMROGRAMAN LINIER DENGAN KOEFISIEN FUNGSI OBJEKTIF, FUNGSI KENDALA DAN VARIABEL KEPUTUSAN BERBENTUK BILANGAN KABUR BESERTA APLIKASINYA
}

\author{
Muhammad Amrullah*
}

\begin{abstract}
In this paper author shall propose an algorithm for solving fuzzy linear programming problems with trapezoidal numbers using a simplex method. In particular, an application of this theory in decision making problems is fuzzy linear programming with a new method for solving fuzzy linear programming problems, by use of rank function. Basically, our method is similar to simplex method that was used for solving linear programming problems in crisp environment before.
\end{abstract}

Keywords: Fuzzy linier programming, rank function, simplex method.

\begin{abstract}
Abstrak
Dalam makalah ini penulis akan mengusulkan algoritma untuk memodelkan masalah pemrograman linear kabur dengan bilangan kabur trapesium menggunakan metode simpleks. Secara khusus dalam aplikasi teori ini adalah masalah pengambilan keputusan pemrograman linear kabur dengan menyajikan metode baru untuk menyelesaikan masalah pemrograman linier kabur dengan menggunakan fungsi ranking. Pada dasarnya, langkahlangkah dalam metode penelitian ini sama dengan dengan metode simpleks yang digunakan untuk memecahkan masalah pemrograman linier tegas.
\end{abstract}

Kata kunci: Fuzzy linear programming, fungsi ranking, metode simpleks.

\section{Pendahuluan}

Logika kabur adalah konsep matematis yang mendasari penalaran kabur yang sangat sederhana, didasarkan pada bahasa alami, memiliki toleransi terhadap data-data yang tepat, fleksibel dan mampu bekerja sama dengan teknik-teknik kendali secara konvensional sehingga mudah dimengerti. Selain itu logika kabur mampu memodelkan fungsi-fungsi non linier yang sangat kompleks serta dapat membangun dan mengaplikasikan pengalaman-pengalaman para pakar secara langsung tanpa harus melalui proses pelatihan. Maka logika kabur merupakan salah satu cara yang tepat untuk memetakan suatu ruang input ke dalam suatu ruang output [7]. Logika kabur pertama kali diperkenalkan oleh Zadeh pada tahun 1965[15], penelitian-penelitian tentang logika kabur dan himpunan kabur berkembang dengan pesat hingga sekarang [1][2][4][5][6][8][11][13][14]. Perkembangan tersebut meliputi aspek teori sampai aspek penerapannya [. Salah satu penerapan himpunan kabur dalam riset operasi adalah masalah pemrograman linier. Masalah pemrograman linier yaitu mencari solusi optimum pada

*Program Stusdi Pascasarjana (S2) Matematika

Fakultas Matematika dan Ilmu Pengetahuan Alam

Universitas Hasanuddin, Makassar, 90245

Email.amrullahambhunk@gmail,.com /amrullahmath4477@gmail.com 


\section{Muhammad Amrullah}

sasaran linier dengan cara menentukan nilai peubah-peubah yang tak diketahui dari batasan liner. Kajian teoritis tentang Fuzzy Linear Programming (FLP) telah banyak dilakukan, diantaranya Stanojevic [12] membahas tentang solusi Fuzzy Linear Programming (FLP) dengan bilangan kabur trapesium. Nasseri pada tahun 2007[9] membahas tentang penggunaan metode simplex untuk menemukan solusi pemrograman linier dengan kendala, koefisien fungsi objektif, dan koefisien fungsi tujuan bernilai kabur. Pandian dan Jayalakshmi pada tahun 2010[10] membahas tentang solusi pemrograman linier dengan variable bersifat kabur, Ebrahimnejad pada tahu 2010 [3] menjelasakan tentang bukti konstruktif dari teori dasar untuk Fuzzy Linear Programming (FLP) dengan variable bersifat kabur.

\section{Bahan dan Metode}

Kurva trapesium pada dasarnya seperti bentuk segitiga, tetapi ada beberapa titik yang memiliki nilai keanggotaan 1 .

$\omega$
$\mu_{\mathrm{A}}(\mathrm{x})$
0

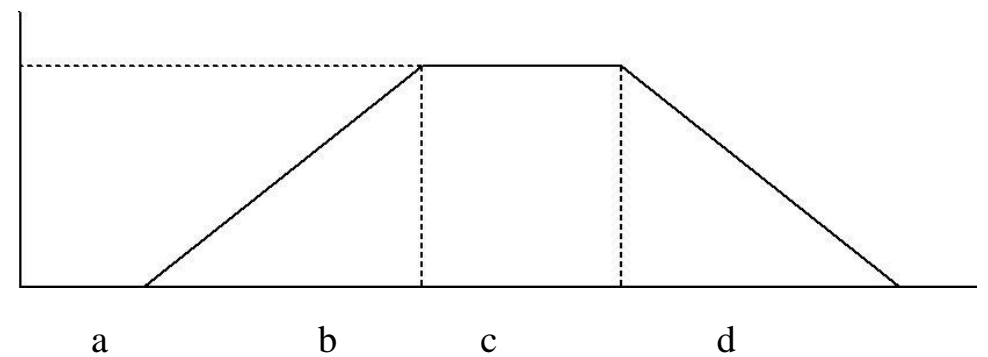

Gambar 2.1 Kurva bilangan kabur trapesium

\section{Definisi II.3}

Menurut Salim dan Molani (2014), semua bilangan kabur A adalah himpunan bagian dari $\mathrm{R}$, yang fungsi keanggotaannya $\mu_{\mathrm{A}}(\mathrm{x})$ memenuhi kondisi berikut :

(i) $\quad \mu_{\mathrm{A}}(\mathrm{x})$ pemetaan dari $\mathrm{R}$ ke interval tertutup $[0, \omega], 0 \leq \omega \leq 1$, adalah kontiniu.

(ii) $\quad \mu_{\mathrm{A}}(\mathrm{x})=0$, untuk semua $\mathrm{x} \in(-\infty, \mathrm{a}]$,

(iii) $\quad \mu_{\mathrm{L}}(\mathrm{x})=\mathrm{L}(\mathrm{x})$ naik pada interval $[\mathrm{a}, \mathrm{b}]$,

(iv) $\quad \mu_{\mathrm{A}}(\mathrm{x})=\omega$, pada interval $[\mathrm{b}, \mathrm{c}]$, dimana $\omega$ konstan dan $0 \leq \omega \leq 1$,

(v) $\quad \mu_{\mathrm{R}}(\mathrm{x})=\mathrm{R}(\mathrm{x})$ turun pada interval $[\mathrm{c}, \mathrm{d}]$,

(vi) $\quad \mu_{\mathrm{A}}(\mathrm{x})=0$, untuk semua $\mathrm{x} \in[\mathrm{d}, \infty)$,

dimana a,b,c,d bilangan Real sedemikian sehingga $\mathrm{a} \leq \mathrm{b} \leq \mathrm{c} \leq \mathrm{d}$.

\section{A. Operasi Bilangan Kabur Trapesium}

\section{A.1 Penjumlahan Bilangan Kabur Trapesium}

Sifat A.1 Jika $\widetilde{\mathrm{A}}_{1}=\left(\mathrm{a}_{1}, \mathrm{~b}_{1}, \mathrm{c}_{1}, \mathrm{~d}_{1}\right) \quad$ dan $\quad \widetilde{\mathrm{A}}_{2}=\left(\mathrm{a}_{2}, \mathrm{~b}_{2}, \mathrm{c}_{2}, \mathrm{~d}_{2}\right)$, maka $\left(\mathrm{a}_{1}+\mathrm{a}_{2}, \mathrm{~b}_{1}+\mathrm{b}_{2}, \mathrm{c}_{1}+\mathrm{c}_{2}, \mathrm{~d}_{1}+\mathrm{d}_{2}\right)$ adalah bentuk bilangan kabur dari $\widetilde{\mathrm{A}}_{1}+\widetilde{\mathrm{A}}_{2}$.

Bukti: dengan transformasi $\mathrm{y}=\mathrm{x}_{1}+\mathrm{x}_{2}$, fungsi keanggotaan bilangan kabur dapat ditulis $\tilde{\mathrm{y}}=\widetilde{\mathrm{A}}_{1}+\widetilde{\mathrm{A}}_{2}$ dengan menggunakan metode potongan $\alpha$. Potongan $\alpha$ sisi kiri dari $\widetilde{\mathrm{A}}_{1}$ untuk $0 \leq \alpha \leq 1$ adalah $\mathrm{X}_{\mathrm{A}_{1} \mathrm{~L}}(\alpha)=\mathrm{a}_{1}+\alpha\left(\mathrm{b}_{1}-\mathrm{a}_{1}\right)$ dan potongan $\alpha$ sisi kanan dari $\widetilde{\mathrm{A}}_{1}$ untuk $0 \leq \alpha \leq 1$ adalah $\mathrm{X}_{\mathrm{A}_{1} \mathrm{R}}(\alpha)=\mathrm{d}_{1}-\alpha\left(\mathrm{d}_{1}-\mathrm{c}_{1}\right)$,

dapat ditulis $\mathrm{x}_{1} \in\left[\mathrm{a}_{1}+\alpha\left(\mathrm{b}_{1}-\mathrm{a}_{1}\right), \mathrm{d}_{1}-\alpha\left(\mathrm{d}_{1}-\mathrm{c}_{1}\right)\right]$,

kemudian, potongan $\alpha$ sisi kiri dari $\widetilde{\mathrm{A}}_{2}$ untuk $0 \leq \alpha \leq 1$ adalah $\mathrm{X}_{\mathrm{A}_{2} \mathrm{~L}}(\alpha)=\mathrm{a}_{2}+\alpha\left(\mathrm{b}_{2}-\mathrm{a}_{2}\right)$, potongan $\alpha$ sisi kanan dari $\widetilde{A}_{2}$ untuk $0 \leq \alpha \leq 1$ adalah $\mathrm{X}_{\mathrm{A}_{2} \mathrm{R}}(\alpha)=\mathrm{d}_{2}-\alpha\left(\mathrm{d}_{2}-\mathrm{c}_{2}\right)$, dapat ditulis $\mathrm{x}_{2} \in\left[\mathrm{a}_{2}+\alpha\left(\mathrm{b}_{2}-\mathrm{a}_{2}\right), \mathrm{d}_{2}-\alpha\left(\mathrm{d}_{2}-\mathrm{c}_{2}\right)\right]$, sehingga, 


\section{Muhammad Amrullah}

$\mathrm{y}=\left(\mathrm{x}_{1}+\mathrm{x}_{2}\right) \in\left[\mathrm{a}_{1}+\mathrm{a}_{2}+\alpha\left(\left(\mathrm{b}_{1}-\mathrm{a}_{1}\right)+\left(\mathrm{b}_{2}-\mathrm{a}_{2}\right)\right), \mathrm{d}_{1}+\mathrm{d}_{2}-\alpha\left(\left(\mathrm{d}_{1}-\mathrm{c}_{1}\right)+\left(\mathrm{d}_{2}-\mathrm{c}_{2}\right)\right)\right]$.

Dari persamaan (1) dapat ditulis

$\alpha=\left(\frac{y-a_{1}-a_{2}}{b_{1}+b_{2}-a_{1}-a_{2}}\right), \quad a_{1}+a_{2} \leq y \leq b_{1}+b_{2}$

dan

$\alpha=\left(\frac{\mathrm{d}_{1}+\mathrm{d}_{2}-\mathrm{y}}{\mathrm{d}_{1}+\mathrm{d}_{2}-\mathrm{c}_{1}-\mathrm{c}_{2}}\right), \quad \mathrm{c}_{1}+\mathrm{c}_{2} \leq \mathrm{y} \leq \mathrm{d}_{1}+\mathrm{d}_{2}$

dari (2) dan (3) diperoleh fungsi keanggotaan $\widetilde{\mathrm{y}}=\widetilde{\mathrm{A}}_{1}+\widetilde{\mathrm{A}}_{2}$ sebagai berikut:

$\mu_{\tilde{y}}(y)=\left\{\begin{array}{cc}\left(\frac{y-a_{1}-a_{2}}{b_{1}+b_{2}-a_{1}-a_{2}}\right), & a_{1}+a_{2} \leq y \leq b_{1}+b_{2} \\ 1 & , b_{1}+b_{2} \leq y \leq c_{1}+c_{2} \\ \left(\frac{d_{1}+d_{2}-y}{d_{1}+d_{2}-c_{1}-c_{2}}\right), & c_{1}+c_{2} \leq y \leq d_{1}+d_{2} \\ 0, & \text { Lainnya }\end{array}\right.$

berdasarkan batasan interval dari fungsi keanggotan (4), terbukti bahwa $\widetilde{\mathrm{A}}_{1}+\widetilde{\mathrm{A}}_{2}=\left(\mathrm{a}_{1}+\mathrm{a}_{2}, \mathrm{~b}_{1}+\mathrm{b}_{2}, \mathrm{c}_{1}+\mathrm{c}_{2}, \mathrm{~d}_{1}+\mathrm{d}_{2}\right)$.

\section{A.2 Pengurangan Bilangan Kabur Trapesium}

Sifat A.2: Jika $\widetilde{A}_{1}=\left(a_{1}, b_{1}, c_{1}, d_{1}\right) \quad$ dan $\quad \widetilde{A}_{2}=\left(a_{2}, b_{2}, c_{2}, d_{2}\right), \quad$ maka $\left(a_{1}-d_{2}, b_{1}-c_{2}, c_{1}-b_{2}, d_{1}-a_{2}\right)$ adalah bentuk bilangan kabur dari $\widetilde{A}_{1}-\widetilde{A}_{2}$.

Bukti : dengan transformasi $\mathrm{y}=\mathrm{x}_{1}-\mathrm{x}_{2}$, fungsi keanggotaan bilangan kabur dapat ditulis $\tilde{y}=\widetilde{\mathrm{A}}_{1}-\widetilde{\mathrm{A}}_{2}$ dengan menggunakan metode potongan $\alpha$. Potongan $\alpha$ sisi kiri dari $\widetilde{\mathrm{A}}_{1}$ untuk $0 \leq \alpha \leq 1$ adalah $\mathrm{X}_{\mathrm{A}_{1} \mathrm{~L}}(\alpha)=\mathrm{a}_{1}+\alpha\left(\mathrm{b}_{1}-\mathrm{a}_{1}\right)$, potongan $\alpha$ sisi kanan dari $\widetilde{\mathrm{A}}_{1}$ untuk $0 \leq \alpha \leq 1$ adalah $\mathrm{X}_{\mathrm{A}_{1} \mathrm{R}}(\alpha)=\mathrm{d}_{1}-\alpha\left(\mathrm{d}_{1}-\mathrm{c}_{1}\right)$, dapat ditulis $\mathrm{x}_{1} \in\left[\mathrm{a}_{1}+\alpha\left(\mathrm{b}_{1}-\mathrm{a}_{1}\right), \mathrm{d}_{1}-\alpha\left(\mathrm{d}_{1}-\mathrm{c}_{1}\right)\right]$,

kemudian, potongan $\alpha$ sisi kiri dari $\widetilde{\mathrm{A}}_{2}$ untuk $0 \leq \alpha \leq 1$ adalah $\mathrm{X}_{\mathrm{A}_{2} \mathrm{~L}}(\alpha)=\mathrm{a}_{2}+\alpha\left(\mathrm{b}_{2}-\mathrm{a}_{2}\right)$, potongan $\alpha$ sisi kanan dari $\widetilde{\mathrm{A}}_{2}$ untuk $0 \leq \alpha \leq 1$ adalah $\mathrm{X}_{\mathrm{A}_{2} \mathrm{R}}(\alpha)=\mathrm{d}_{2}-\alpha\left(\mathrm{d}_{2}-\mathrm{c}_{2}\right)$,

dapat ditulis $\mathrm{x}_{2} \in\left[\mathrm{a}_{2}+\alpha\left(\mathrm{b}_{2}-\mathrm{a}_{2}\right), \mathrm{d}_{2}-\alpha\left(\mathrm{d}_{2}-\mathrm{c}_{2}\right)\right]$,

sehingga, $y=\left(\mathrm{x}_{1}-\mathrm{x}_{2}\right) \in \begin{aligned} & {\left[\mathrm{a}_{1}-\mathrm{d}_{2}+\alpha\left(\left(\mathrm{b}_{1}-\mathrm{a}_{1}\right)+\left(\mathrm{d}_{2}-\mathrm{c}_{2}\right)\right) \text {, }\right.} \\ & \left.\mathrm{d}_{1}-\mathrm{a}_{2}-\alpha\left(\left(\mathrm{d}_{1}-\mathrm{c}_{1}\right)+\left(\mathrm{b}_{2}-\mathrm{a}_{2}\right)\right)\right]\end{aligned}$

dari persamaan (5) dapat ditulis

$$
\left.d_{1}-a_{2}-\alpha\left(\left(d_{1}-c_{1}\right)+\left(b_{2}-a_{2}\right)\right)\right]
$$

dan

$\alpha=\left(\frac{\mathrm{d}_{1}-\mathrm{a}_{2}-\mathrm{y}}{\mathrm{b}_{2}+\mathrm{d}_{1}-\mathrm{c}_{1}-\mathrm{a}_{2}}\right), \quad \mathrm{c}_{1}-\mathrm{b}_{2} \leq \mathrm{y} \leq \mathrm{d}_{1}-\mathrm{a}_{2}$

dari (6) dan (7) diperoleh fungsi keanggotaan $\tilde{y}=\widetilde{A}_{1}-\widetilde{A}_{2}$ sebagai berikut:

$\mu_{\tilde{y}}(y)=\left\{\begin{array}{cc}\left(\frac{y-a_{1}+d_{2}}{b_{1}+d_{2}-a_{1}-c_{2}}\right), & a_{1}-d_{2} \leq y \leq b_{1}-c_{2} \\ 1, & , b_{1}-c_{2} \leq y \leq c_{1}-b_{2} \\ \left(\frac{d_{1}-a_{2}-y}{b_{2}+d_{1}-c_{1}-a_{2}}\right), & c_{1}-b_{2} \leq y \leq d_{1}-a_{2} \\ 0, & \text { lainnya }\end{array}\right.$

berdasarkan batasan interval dari fungsi keanggotan (8) maka terbukti $\widetilde{\mathrm{A}}_{1}-\widetilde{\mathrm{A}}_{2}=\left(\mathrm{a}_{1}-\mathrm{d}_{2}, \mathrm{~b}_{1}-\mathrm{c}_{2}, \mathrm{c}_{1}-\mathrm{b}_{2}, \mathrm{~d}_{1}-\mathrm{a}_{2}\right)$.

\section{A.3 Perkalian Bilangan Kabur}

Sifat A.3: Jika $\widetilde{A}_{1}=\left(a_{1}, b_{1}, c_{1}, d_{1}\right)$ dan $\widetilde{A}_{2}=\left(a_{2}, b_{2}, c_{2}, d_{2}\right)$, maka $\left(a_{1} a_{2}, b_{1} b_{2}, c_{1} c_{2}, d_{1} d_{2}\right)$ adalah bentuk bilangan kabur dari $\widetilde{A}_{1} \times \widetilde{A}_{2}$. 


\section{Muhammad Amrullah}

Bukti: dengan tranformasi $\mathrm{y}=\mathrm{x}_{1} \mathrm{x}_{2}$, fungsi keanggotaan bilangan kabur dapat ditulis $\tilde{\mathrm{y}}=\widetilde{\mathrm{A}}_{1} \times \widetilde{\mathrm{A}}_{2}$ dengan menggunakan metode potongan $\alpha$.

Potongan $\alpha$ sisi kiri dari $\widetilde{\mathrm{A}}_{1}$ untuk $0 \leq \alpha \leq 1$ adalah $\mathrm{X}_{\mathrm{A}_{1} \mathrm{~L}}(\alpha)=\mathrm{a}_{1}+\alpha\left(\mathrm{b}_{1}-\mathrm{a}_{1}\right)$, potongan$\alpha$ sisi kanan dari $\widetilde{\mathrm{A}}_{1}$ untuk $0 \leq \alpha \leq 1$ adalah $\mathrm{X}_{\mathrm{A}_{1} \mathrm{R}}(\alpha)=\mathrm{d}_{1}-\alpha\left(\mathrm{d}_{1}-\mathrm{c}_{1}\right)$, dapat ditulis $\mathrm{x}_{1} \in\left[\mathrm{a}_{1}+\alpha\left(\mathrm{b}_{1}-\mathrm{a}_{1}\right), \mathrm{d}_{1}-\alpha\left(\mathrm{d}_{1}-\mathrm{c}_{1}\right)\right]$, kemudian, potongan $\alpha$ sisi kiri dari $\widetilde{\mathrm{A}}_{2}$ untuk $0 \leq \alpha \leq 1$ adalah $\mathrm{X}_{\mathrm{A}_{2} \mathrm{~L}}(\alpha)=\mathrm{a}_{2}+\alpha\left(\mathrm{b}_{2}-\mathrm{a}_{2}\right)$, potongan $\alpha$ sisi kanan dari $\widetilde{\mathrm{A}}_{2}$ untuk $0 \leq \alpha \leq 1$ adalah $\mathrm{X}_{\mathrm{A}_{2} \mathrm{R}}(\alpha)=\mathrm{d}_{2}-\alpha\left(\mathrm{d}_{2}-\mathrm{c}_{2}\right)$,

dapat ditulis $\mathrm{x}_{2} \in\left[\mathrm{a}_{2}+\alpha\left(\mathrm{b}_{2}-\mathrm{a}_{2}\right), \mathrm{d}_{2}-\alpha\left(\mathrm{d}_{2}-\mathrm{c}_{2}\right)\right]$,

sehingga,

$y=x_{1} x_{2} \in \begin{aligned} & {\left[a_{1} a_{2}+\alpha^{2}\left(b_{1}-a_{1}\right)\left(b_{2}-a_{2}\right)+\alpha\left(a_{1}\left(b_{2}-a_{2}\right)+a_{2}\left(b_{1}-a_{1}\right)\right),\right.} \\ & \left.d_{1} d_{2}+\alpha^{2}\left(d_{1}-c_{1}\right)\left(d_{2}-c_{2}\right)-\alpha\left(d_{1}\left(d_{2}-c_{2}\right)+d_{2}\left(d_{1}-c_{1}\right)\right)\right]\end{aligned}$

dari persamaan (9) dapat ditulis

$\alpha=\left(\frac{-B+\sqrt{B^{2}-4 A C}}{2 A}\right), \quad a_{1} a_{2} \leq y \leq b_{1} b_{2}$

dan

$\alpha=\left(\frac{-B^{\prime}+\sqrt{B^{2}-4 A^{\prime} C^{\prime}}}{2 A^{\prime}}\right), \quad c_{1} c_{2} \leq y \leq d_{1} d_{2}$

dari (10) dan (11) diperoleh fungsi keanggotaan $\tilde{\mathrm{y}}=\widetilde{\mathrm{A}}_{1} \times \widetilde{\mathrm{A}}_{2}$ sebagai berikut

$\mu(y)=\left\{\begin{array}{cl}\left(\frac{-B^{+} \sqrt{B^{2}-4 A C}}{2 A}\right), & a_{1} a_{2} \leq y \leq b_{1} b_{2}, \\ 1, & b_{1} b_{2} \leq y \leq c_{1} c_{2} \\ \left(\frac{-B^{\prime}-\sqrt{B^{2}-4 A^{\prime} C^{\prime}}}{2 A^{\prime}}\right), & c_{1} c_{2} \leq y \leq d_{1} d_{2} \\ 0, & \text { lainnya }\end{array}\right.$

dimana

$A=\left(b_{1}-a_{1}\right)\left(b_{2}-a_{2}\right)$,

$\mathrm{B}=\mathrm{a}_{1}\left(\mathrm{~b}_{2}-\mathrm{a}_{2}\right)+\mathrm{a}_{2}\left(\mathrm{~b}_{1}-\mathrm{a}_{1}\right)$,

$\mathrm{C}=\mathrm{a}_{1} \mathrm{a}_{2}-\mathrm{y}$,

$A^{\prime}=\left(d_{1}-c_{1}\right)\left(d_{2}-c_{2}\right)$,

$B^{\prime}=-\left\{d_{1}\left(d_{2}-c_{2}\right)+d_{2}\left(d_{1}-c_{1}\right)\right\}$ dan

$\mathrm{C}^{\prime}=\mathrm{d}_{1} \mathrm{~d}_{2}-\mathrm{y}$

berdasarkan batasan interval dari fungsi keanggotan (12), terbukti bahwa $\widetilde{\mathrm{A}}_{1} \times \widetilde{\mathrm{A}}_{2}=$ $\left(a_{1} a_{2}, b_{1} b_{2}, c_{1} c_{2}, d_{1} d_{2}\right)$.

\section{A.4 Perkalian Skalar Pada Bilangan Kabur}

Sifat A.4:

(a) Jika $\tilde{u}=\left(u_{1}, u_{2}, u_{3}, u_{4} ; w\right)$ dan $y=k u, k>0$ maka $\left(k u_{1}, k u_{2}, k u_{3}, k u_{4}\right)$ adalah bilangan kabur dari $\tilde{y}=k \tilde{u}$.

(b) Jika $\tilde{u}=\left(u_{1}, u_{2}, u_{3}, u_{4} ; w\right)$ dan $y=k u, k<0$ maka $\left(k u_{4}, k u_{3}, k u_{2}, k u_{1}\right)$ adalah bilangan kabur dari $\tilde{y}=k \tilde{u}$.

Bukti:

(a) Jika $\mathrm{k}>0$, dengan transformasi $\mathrm{y}=\mathrm{ku}$, dapat ditulis dalam fungsi keanggotaan himpunan kabur $\mathrm{y}=\mathrm{ku}$ dengan menggunakan metode potongan $\alpha$. Potongan $\alpha$ sisi kiri dari ũ untuk $0 \leq \alpha \leq 1$ adalah $\mathrm{X}_{\mathrm{UL}}(\alpha)=\mathrm{u}_{1}+\alpha\left(\mathrm{u}_{2}-\mathrm{u}_{1}\right)$, potongan $\alpha$ sisi kanan dari 


\section{Muhammad Amrullah}

ũ untuk $0 \leq \alpha \leq 1$ adalah $\quad \mathrm{X}_{\mathrm{UR}}(\alpha)=\mathrm{u}_{4}-\alpha\left(\mathrm{u}_{4}-\mathrm{u}_{3}\right), \quad$ dapat ditulis $\mathrm{u} \in\left[\mathrm{u}_{1}+\alpha\left(\mathrm{u}_{2}-\mathrm{u}_{1}\right), \mathrm{u}_{4}-\alpha\left(\mathrm{u}_{4}-\mathrm{u}_{3}\right)\right]$,

kemudian

$\mathrm{y}=\mathrm{ku} \in\left[\mathrm{ku}_{1}+\alpha\left(\mathrm{ku}_{2}-\mathrm{ku}_{1}\right), \mathrm{ku}_{4}-\alpha\left(\mathrm{ku}_{4}-\mathrm{ku}_{3}\right)\right]$,

sehingga

$\alpha=\left(\frac{\mathrm{y}-\mathrm{ku}_{1}}{\mathrm{ku}_{2}-\mathrm{ku}_{1}}\right), \quad \mathrm{ku}_{1} \leq \mathrm{y} \leq \mathrm{ku}_{2}$

dan

$\alpha=\left(\frac{\mathrm{ku}_{4}-\mathrm{y}}{\mathrm{ku}_{4}-\mathrm{ku}_{3}}\right), \quad \mathrm{ku}_{3} \leq \mathrm{y} \leq \mathrm{ku}_{4}$.

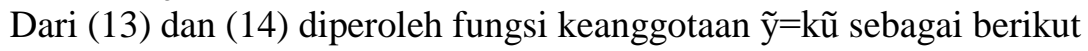

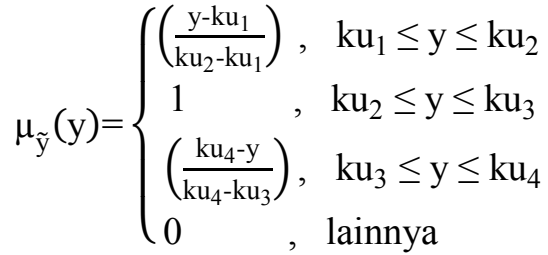

(b) Dengan cara yang sama, jika $\mathrm{y}=\mathrm{ku}, \mathrm{k}<0$ maka

$\mu_{\tilde{y}}(y)=\left\{\begin{array}{cl}\left(\frac{k u_{4}-\mathrm{y}}{\mathrm{ku}_{4}-\mathrm{ku}_{3}}\right), & \mathrm{ku}_{4} \leq \mathrm{y} \leq \mathrm{ku}_{3} \\ 1, & \mathrm{ku}_{3} \leq \mathrm{y} \leq \mathrm{ku}_{2} \\ \left(\frac{\mathrm{y}-\mathrm{ku}_{1}}{\mathrm{ku}_{2}-\mathrm{ku}_{1}}\right), & \mathrm{ku}_{2} \leq \mathrm{y} \leq \mathrm{ku}_{1} \\ 0 & , \text { lainnya }\end{array}\right.$

berdasarkan batasan interval dari fungsi keanggotan (15) dan (16), terbukti bahwa

(a) $\mathrm{ku}=\left(\mathrm{ku}_{1}, \mathrm{ku}_{2}, \mathrm{ku}_{3}, \mathrm{ku}_{4}\right)$, dan

(b) $\mathrm{ku}=\left(\mathrm{ku}_{4}, \mathrm{ku}_{3}, \mathrm{ku}_{2}, \mathrm{ku}_{1}\right), \mathrm{k}<0$

\section{A.5 Pembagian Bilangan Kabur}

Sifat A.5: Jika $\widetilde{A}_{1}=\left(a_{1}, b_{1}, c_{1}, d_{1}\right) \quad$ dan $\quad \widetilde{A}_{2}=\left(a_{2}, b_{2}, c_{2}, d_{2}\right), \quad$ maka $\left(a_{1} / d_{2}, b_{1} / c_{2}, c_{1} / b_{2}, d_{1} / a_{2}\right)$ adalah bentuk bilangan kabur dari $\widetilde{A}_{1} / \widetilde{A}_{2}$

Bukti: dengan transformasi $\mathrm{y}=\mathrm{x}_{1} / \mathrm{x}_{2}$, dapat ditulis dalam fungsi keanggotaan himpunan kabur $\tilde{\mathrm{y}}=\widetilde{\mathrm{A}}_{1} / \widetilde{\mathrm{A}}_{2}$ dengan metode potongan $\alpha$.

Potongan $\alpha$ sisi kiri dari $\widetilde{\mathrm{A}}_{1}$ untuk $0 \leq \alpha \leq 1$ adalah $\mathrm{X}_{\mathrm{A}_{1} \mathrm{~L}}(\alpha)=\mathrm{a}_{1}+\alpha\left(\mathrm{b}_{1}-\mathrm{a}_{1}\right)$, potongan $\alpha$ sisi kanan dari $\widetilde{\mathrm{A}}_{1}$ untuk $0 \leq \alpha \leq 1$ adalah $\mathrm{X}_{\mathrm{A}_{1} \mathrm{R}}(\alpha)=\mathrm{d}_{1}-\alpha\left(\mathrm{d}_{1}-\mathrm{c}_{1}\right)$,

dapat ditulis $\mathrm{x}_{1} \in\left[\mathrm{a}_{1}+\alpha\left(\mathrm{b}_{1}-\mathrm{a}_{1}\right), \mathrm{d}_{1}-\alpha\left(\mathrm{d}_{1}-\mathrm{c}_{1}\right)\right]$,

kemudian,

potongan $\alpha$ sisi kiri dari $\widetilde{\mathrm{A}}_{2}$ untuk $0 \leq \alpha \leq 1$ adalah $\mathrm{X}_{\mathrm{A}_{2} \mathrm{~L}}(\alpha)=\mathrm{a}_{2}+\alpha\left(\mathrm{b}_{2}-\mathrm{a}_{2}\right)$, potongan$\alpha$ sisi kanan dari $\widetilde{\mathrm{A}}_{2}$ untuk $0 \leq \alpha \leq 1$ adalah $\mathrm{X}_{\mathrm{A}_{2} \mathrm{R}}(\alpha)=\mathrm{d}_{2}-\alpha\left(\mathrm{d}_{2}-\mathrm{c}_{2}\right)$, dapat ditulis $\mathrm{x}_{2} \in\left[\mathrm{a}_{2}+\alpha\left(\mathrm{b}_{2}-\mathrm{a}_{2}\right), \mathrm{d}_{2}-\alpha\left(\mathrm{d}_{2}-\mathrm{c}_{2}\right)\right]$,

kemudian,

$\mathrm{y}=\mathrm{x}_{1} / \mathrm{x}_{2} \in \frac{\left[\mathrm{a}_{1}+\alpha\left(\mathrm{b}_{1}-\mathrm{a}_{1}\right), \mathrm{d}_{1}-\alpha\left(\mathrm{d}_{1}-\mathrm{c}_{1}\right)\right]}{\left[\mathrm{a}_{2}+\alpha\left(\mathrm{b}_{2}-\mathrm{a}_{2}\right), \mathrm{d}_{2}-\alpha\left(\mathrm{d}_{2}-\mathrm{c}_{2}\right)\right]}$,

$y=x_{1} / x_{2} \in\left[\frac{a_{1}+\alpha\left(b_{1}-a_{1}\right)}{d_{2}-\alpha\left(d_{2}-c_{2}\right)}, \frac{d_{1}-\alpha\left(d_{1}-c_{1}\right)}{a_{2}+\alpha\left(b_{2}-a_{2}\right)}\right]$

sehingga

$y=\frac{a_{1}+\alpha\left(b_{1}-a_{1}\right)}{d_{2}-\alpha\left(d_{2}-c_{2}\right)}$

$\Rightarrow \alpha=\left(\frac{\mathrm{d}_{2} \mathrm{y}-\mathrm{a}_{1}}{\left(\mathrm{~b}_{1}-\mathrm{a}_{1}\right)+\mathrm{y}\left(\mathrm{d}_{2}-\mathrm{c}_{2}\right)}\right), \mathrm{a}_{1} / \mathrm{d}_{2} \leq \mathrm{y} \leq \mathrm{b}_{1} / \mathrm{c}_{2}$

dan 


\section{Muhammad Amrullah}

$y=\frac{d_{1}-\alpha\left(d_{1}-c_{1}\right)}{a_{2}+\alpha\left(b_{2}-a_{2}\right)}$

$\Rightarrow \alpha=\left(\frac{\mathrm{d}_{1}-\mathrm{a}_{2} \mathrm{y}}{\mathrm{y}\left(\mathrm{b}_{2}-\mathrm{a}_{2}\right)+\left(\mathrm{d}_{1}-\mathrm{c}_{1}\right)}\right), \mathrm{c}_{1} / \mathrm{b}_{2} \leq \mathrm{y} \leq \mathrm{d}_{1} / \mathrm{a}_{2}$

dari (18) dan (19) diperoleh fungsi keanggotaan dari $\tilde{y}=\widetilde{\mathrm{A}}_{1} / \widetilde{\mathrm{A}}_{2}$ sebagai berikut

$\mu(y)=\left\{\begin{array}{cc}\left(\frac{d_{2} y-a_{1}}{\left(b_{1}-a_{1}\right)+y\left(d_{2}-c_{2}\right)}\right), & a_{1} / d_{2} \leq y \leq b_{1} / c_{2} \\ 1 & b_{1} / c_{2} \leq y \leq c_{1} / b_{2} \\ \left(\frac{d_{1}-a_{2} y}{y\left(b_{2}-a_{2}\right)+\left(d_{1}-c_{1}\right)}\right), & c_{1} / b_{2} \leq y \leq d_{1} / a_{2} \\ 0 & \text { lainnya }\end{array}\right.$

berdasarkan batasan interval dari fungsi keanggotan (20), maka terbukti bahwa $\widetilde{\mathrm{A}}_{1} / \widetilde{\mathrm{A}}_{2}=\left(\mathrm{a}_{1} / \mathrm{d}_{2}, \mathrm{~b}_{1} / \mathrm{c}_{2}, \mathrm{c}_{1} / \mathrm{b}_{2}, \mathrm{~d}_{1} / \mathrm{a}_{2}\right)$.

\section{B. Fungsi Ranking Bilangan Kabur Trapesium}

Sifat A6. Jika $R(\widetilde{A})=\frac{1}{2} \int_{0}^{1}\left(\right.$ inf $\left.\widetilde{A}_{\lambda}+\sup \widetilde{A}_{\lambda}\right) d \lambda \quad$ adalah bentuk tegas dari bilangan kabur $\widetilde{A}=(a, b, c, d)$, maka berlaku $R(\widetilde{A})=\frac{1}{2}\left(m_{1}+n_{1}+\frac{1}{2}\left(\beta_{1}-\alpha_{1}\right)\right)$, dimana $m_{1}=b, m_{2}=c, \beta_{1}=d-c$ dan $\alpha_{1}=b-a$.

Bukti : misalkan $\inf \widetilde{\mathrm{A}}_{\lambda}=\mathrm{L}^{-1}(\mathrm{x})$ adalah sisi kiri dari bilangan kabur trapesium, $\sup \widetilde{\mathrm{A}}_{\lambda}=\mathrm{R}^{-1}(\mathrm{x})$ adalah sisi kanan bilangan kabur dan $\widetilde{\mathrm{A}}=(\mathrm{a}, \mathrm{b}, \mathrm{c}, \mathrm{d})$ adalah bentuk umum bilangan kabur trapesium, dapat ditulis $\mathrm{L}(\mathrm{x})=\frac{\mathrm{x}-\mathrm{a}}{\mathrm{b}-\mathrm{a}}, \Rightarrow \mathrm{L}^{-1}(\mathrm{x})=(\mathrm{b}-\mathrm{a}) \mathrm{x}+\mathrm{a}$ dan $\mathrm{R}(\mathrm{x})=\frac{\mathrm{d}-\mathrm{x}}{\mathrm{d}-\mathrm{c}} \mathrm{R}^{-1}(\mathrm{x})=(\mathrm{c}-\mathrm{d}) \mathrm{x}+\mathrm{d}$, sehingga $R(\widetilde{A})=\frac{1}{2} \int_{0}^{1}\left(\inf \widetilde{A}_{\lambda}+\sup \widetilde{A}_{\lambda}\right) d \lambda=\frac{1}{2} \int_{0}^{1}((b-a) x+a+(c-d) x+d) d x$

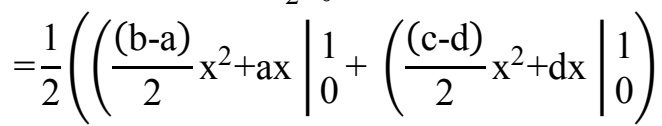
$=\frac{1}{2}\left(\left(\frac{(\mathrm{b}-\mathrm{a})}{2}+\mathrm{a}\right)+\left(\frac{(\mathrm{c}-\mathrm{d})}{2}+\mathrm{d}\right)\right)$

$=\frac{1}{2}\left(\frac{\mathrm{a}+\mathrm{b}+\mathrm{c}+\mathrm{d}}{2}\right)$

$=\frac{1}{2}\left(\left(\frac{2 b-(b-a)}{2}\right)+\left(\frac{2 c+(d-c)}{2}\right)\right)$

$=\frac{1}{2}\left(\mathrm{~b}+\mathrm{c}+\frac{1}{2}((\mathrm{~d}-\mathrm{c})-(\mathrm{b}-\mathrm{a}))\right)$

$=\frac{1}{2}\left(\mathrm{~m}_{1}+\mathrm{n}_{1}+\frac{1}{2}\left(\beta_{1}-\alpha_{1}\right)\right)$

sehingga terbukti $\mathrm{R}(\widetilde{\mathrm{A}})=\frac{1}{2} \int_{0}^{1}\left(\inf \widetilde{\mathrm{A}}_{\lambda}+\sup \widetilde{\mathrm{A}}_{\lambda}\right) \mathrm{d} \lambda=\frac{1}{2}\left(\mathrm{~m}_{1}+\mathrm{n}_{1}+\frac{1}{2}\left(\beta_{1}-\alpha\right)\right)$.

\section{HASIL PENELITIAN}

Diberikan fungsi kendala dan fungsi obyektif sebagai berikut:

$\operatorname{Max} \widetilde{Z}=\left(\mathrm{u}_{1}, \mathrm{u}_{2}, \mathrm{u}_{3}, \mathrm{u}_{4}\right)\left(\mathrm{x}_{11}, \mathrm{x}_{12}, \mathrm{x}_{13}, \mathrm{x}_{14}\right)+\left(\mathrm{v}_{1}, \mathrm{v}_{2}, \mathrm{v}_{3}, \mathrm{v}_{4}\right)\left(\mathrm{x}_{21}, \mathrm{x}_{22}, \mathrm{x}_{23}, \mathrm{x}_{24}\right)$

dengan kendala,

$\left(\mathrm{x}_{1}, \mathrm{x}_{2}, \mathrm{x}_{3}, \mathrm{x}_{4}\right)\left(\mathrm{x}_{11}, \mathrm{x}_{12}, \mathrm{x}_{13}, \mathrm{x}_{14}\right)+\left(\mathrm{y}_{1}, \mathrm{y}_{2}, \mathrm{y}_{3}, \mathrm{y}_{4}\right)\left(\mathrm{x}_{21}, \mathrm{x}_{22}, \mathrm{x}_{23}, \mathrm{x}_{24}\right) \leq$

$\left(\mathrm{z}_{1}, \mathrm{z}_{2}, \mathrm{z}_{3}, \mathrm{z}_{4}\right)$

$\left(\mathrm{p}_{1}, \mathrm{p}_{2}, \mathrm{p}_{3}, \mathrm{p}_{4}\right)\left(\mathrm{x}_{11}, \mathrm{x}_{12}, \mathrm{x}_{13}, \mathrm{x}_{14}\right)+\left(\mathrm{q}_{1}, \mathrm{q}_{2}, \mathrm{q}_{3}, \mathrm{q}_{4}\right)\left(\mathrm{x}_{21}, \mathrm{x}_{22}, \mathrm{x}_{23}, \mathrm{x}_{24}\right) \leq$

$\left(\mathrm{r}_{1}, \mathrm{r}_{2}, \mathrm{r}_{3}, \mathrm{r}_{4}\right)$.

Diasumsikan variabel keputusan $\left(\mathrm{x}_{11}, \mathrm{x}_{12}, \mathrm{x}_{13}, \mathrm{x}_{14}\right)$ dan $\left(\mathrm{x}_{21}, \mathrm{x}_{22}, \mathrm{x}_{23}, \mathrm{x}_{24}\right)$ adalah variabel yang bersifat kabur, dimana $\mathrm{R}\left(\mathrm{x}_{11}, \mathrm{x}_{12}, \mathrm{x}_{13}, \mathrm{x}_{14}\right)$ dan $\mathrm{R}\left(\mathrm{x}_{21}, \mathrm{x}_{22}, \mathrm{x}_{23}, \mathrm{x}_{24}\right)$ adalah 
bentuk tegas dari keduanya, maka berlaku $\tilde{\mathrm{x}}_{1}=\mathrm{R}\left(\mathrm{x}_{11}, \mathrm{x}_{12}, \mathrm{x}_{13}, \mathrm{x}_{14}\right)$ dan $\tilde{\mathrm{x}}_{2}=\mathrm{R}\left(\mathrm{x}_{21}, \mathrm{x}_{22}\right.$, $\mathrm{x}_{23}, \mathrm{x}_{24}$ ) sehingga fungsi kendala dan fungsi obyektif dapat ditulis sebagai berikut:

$\operatorname{Max} \widetilde{Z}=\left(u_{1}, u_{2}, u_{3}, u_{4}\right) \tilde{x}_{1}+\left(v_{1}, v_{2}, v_{3}, v_{4}\right) \tilde{x}_{2}$

Dengan kendala,

$\left(\mathrm{x}_{1}, \mathrm{x}_{2}, \mathrm{x}_{3}, \mathrm{x}_{4}\right) \tilde{\mathrm{x}}_{1}+\left(\mathrm{y}_{1}, \mathrm{y}_{2}, \mathrm{y}_{3}, \mathrm{y}_{4}\right) \tilde{\mathrm{x}}_{2} \leq\left(\mathrm{z}_{1}, \mathrm{z}_{2}, \mathrm{z}_{3}, \mathrm{z}_{4}\right)$

$\left(\mathrm{p}_{1}, \mathrm{p}_{2}, \mathrm{p}_{3}, \mathrm{p}_{4}\right) \tilde{\mathrm{x}}_{1}+\left(\mathrm{q}_{1}, \mathrm{q}_{2}, \mathrm{q}_{3}, \mathrm{q}_{4}\right) \tilde{\mathrm{x}}_{2} \leq\left(\mathrm{r}_{1}, \mathrm{r}_{2}, \mathrm{r}_{3}, \mathrm{r}_{4}\right)$

berdasarkan sifat A.4, maka fungsi kendala dan fungsi obyektif menjadi :

$\operatorname{Max} \mathrm{Z}_{1}=\left(\mathrm{u}_{1}\right) \tilde{\mathrm{x}}_{1}+\left(\mathrm{v}_{1}\right) \tilde{\mathrm{x}}_{2}$

$\left(\mathrm{x}_{1}, \mathrm{x}_{2}, \mathrm{x}_{3}, \mathrm{x}_{4}\right) \tilde{\mathrm{x}}_{1}+\left(\mathrm{y}_{1}, \mathrm{y}_{2}, \mathrm{y}_{3}, \mathrm{y}_{4}\right) \tilde{\mathrm{x}}_{2} \leq\left(\mathrm{z}_{1}, \mathrm{z}_{2}, \mathrm{z}_{3}, \mathrm{z}_{4}\right)$

$\left(\mathrm{p}_{1}, \mathrm{p}_{2}, \mathrm{p}_{3}, \mathrm{p}_{4}\right) \tilde{\mathrm{x}}_{1}+\left(\mathrm{q}_{1}, \mathrm{q}_{2}, \mathrm{q}_{3}, \mathrm{q}_{4}\right) \tilde{\mathrm{x}}_{2} \leq\left(\mathrm{r}_{1}, \mathrm{r}_{2}, \mathrm{r}_{3}, \mathrm{r}_{4}\right)$

$\operatorname{Max} Z_{2}=\left(\mathrm{u}_{2}\right) \tilde{\mathrm{x}}_{1}+\left(\mathrm{v}_{2}\right) \tilde{\mathrm{x}}_{2}$

$\left(\mathrm{x}_{1}, \mathrm{x}_{2}, \mathrm{x}_{3}, \mathrm{x}_{4}\right) \tilde{\mathrm{x}}_{1}+\left(\mathrm{y}_{1}, \mathrm{y}_{2}, \mathrm{y}_{3}, \mathrm{y}_{4}\right) \tilde{\mathrm{x}}_{2} \leq\left(\mathrm{z}_{1}, \mathrm{z}_{2}, \mathrm{z}_{3}, \mathrm{z}_{4}\right)$

$\left(\mathrm{p}_{1}, \mathrm{p}_{2}, \mathrm{p}_{3}, \mathrm{p}_{4}\right) \tilde{\mathrm{x}}_{1}+\left(\mathrm{q}_{1}, \mathrm{q}_{2}, \mathrm{q}_{3}, \mathrm{q}_{4}\right) \tilde{\mathrm{x}}_{2} \leq\left(\mathrm{r}_{1}, \mathrm{r}_{2}, \mathrm{r}_{3}, \mathrm{r}_{4}\right)$

$\operatorname{Max} \mathrm{Z}_{3}=\left(\mathrm{u}_{3}\right) \tilde{\mathrm{x}}_{1}+\left(\mathrm{v}_{3}\right) \tilde{\mathrm{x}}_{2}$

$\left(\mathrm{x}_{1}, \mathrm{x}_{2}, \mathrm{x}_{3}, \mathrm{x}_{4}\right) \tilde{\mathrm{x}}_{1}+\left(\mathrm{y}_{1}, \mathrm{y}_{2}, \mathrm{y}_{3}, \mathrm{y}_{4}\right) \tilde{\mathrm{x}}_{2} \leq\left(\mathrm{z}_{1}, \mathrm{z}_{2}, \mathrm{z}_{3}, \mathrm{z}_{4}\right)$

$\left(\mathrm{p}_{1}, \mathrm{p}_{2}, \mathrm{p}_{3}, \mathrm{p}_{4}\right) \tilde{\mathrm{x}}_{1}+\left(\mathrm{q}_{1}, \mathrm{q}_{2}, \mathrm{q}_{3}, \mathrm{q}_{4}\right) \tilde{\mathrm{x}}_{2} \leq\left(\mathrm{r}_{1}, \mathrm{r}_{2}, \mathrm{r}_{3}, \mathrm{r}_{4}\right)$

$\operatorname{Max} \mathrm{Z}_{4}=\left(\mathrm{u}_{4}\right) \tilde{\mathrm{x}}_{1}+\left(\mathrm{v}_{4}\right) \tilde{\mathrm{x}}_{2}$

$\left(\mathrm{x}_{1}, \mathrm{x}_{2}, \mathrm{x}_{3}, \mathrm{x}_{4}\right) \tilde{\mathrm{x}}_{1}+\left(\mathrm{y}_{1}, \mathrm{y}_{2}, \mathrm{y}_{3}, \mathrm{y}_{4}\right) \tilde{\mathrm{x}}_{2} \leq\left(\mathrm{z}_{1}, \mathrm{z}_{2}, \mathrm{z}_{3}, \mathrm{z}_{4}\right)$

$\left(\mathrm{p}_{1}, \mathrm{p}_{2}, \mathrm{p}_{3}, \mathrm{p}_{4}\right) \tilde{\mathrm{x}}_{1}+\left(\mathrm{q}_{1}, \mathrm{q}_{2}, \mathrm{q}_{3}, \mathrm{q}_{4}\right) \tilde{\mathrm{x}}_{2} \leq\left(\mathrm{r}_{1}, \mathrm{r}_{2}, \mathrm{r}_{3}, \mathrm{r}_{4}\right)$,

dari (21), (22), (23) dan (24) kemudian diselesaikan menggunakan metode simplex dengan langkah-langkah sebagai berikut :

\section{Langkah 1}

Menentukan pemecahan dasar awal yang layak dengan menggunakan bentuk standar (dengan sisi kanan semua non negatif)

Sehingga menjadi:

Tabel 4.1 Bentuk Awal 1

\begin{tabular}{|c|c|c|c|c|c|}
\hline & $\tilde{\mathrm{x}}_{1}$ & $\tilde{\mathrm{x}}_{2}$ & $\tilde{\mathrm{x}}_{3}$ & $\tilde{\mathrm{x}}_{4}$ & $\mathrm{NK}$ \\
\hline $\mathrm{Z}_{\mathrm{n}}$ & $-\left(\mathrm{u}_{\mathrm{n}}\right)$ & $-\left(\mathrm{v}_{\mathrm{n}}\right)$ & $(0)$ & $(0)$ & $(0)$ \\
\hline$\tilde{\mathrm{x}}_{3}$ & $\left(\mathrm{x}_{1}, \mathrm{x}_{2}, \mathrm{x}_{3}, \mathrm{x}_{4}\right)$ & $\left(\mathrm{y}_{1}, \mathrm{y}_{2}, \mathrm{y}_{3}, \mathrm{y}_{4}\right)$ & $(1,1,1,1)$ & $(0,0,0,0)$ & $\left(\mathrm{z}_{1}, \mathrm{z}_{2}, \mathrm{z}_{3}, \mathrm{z}_{4}\right)$ \\
\hline$\tilde{\mathrm{x}}_{4}$ & $\left(\mathrm{p}_{1}, \mathrm{p}_{2}, \mathrm{p}_{3}, \mathrm{p}_{4}\right)$ & $\left(\mathrm{q}_{1}, \mathrm{q}_{2}, \mathrm{q}_{3}, \mathrm{q}_{4}\right)$ & $(0,0,0,0)$ & $(1,1,1,1)$ & $\left(\mathrm{r}_{1}, \mathrm{r}_{2}, \mathrm{r}_{3}, \mathrm{r}_{4}\right)$ \\
\hline
\end{tabular}

dimana $n=1,2,3$ dan 4

\section{Langkah 2}

Menentukan variabel kolom kunci (variabel non dasar dengan koefisien yang paling negatif), yang mana variabel sekolomnya akan menjadi kolom kunci.

Misalkan nilai $\left(-\mathrm{v}_{\mathrm{n}}\right)$ merupakan variabel yang memiliki koefisien paling negatif, sehingga kolom $\tilde{\mathrm{X}}_{2}$ menjadi kolom kunci.

Tabel 4.2 Kolom Kunci 1

\begin{tabular}{|c|c|c|c|c|c|}
\hline & $\tilde{\mathrm{x}}_{1}$ & $\tilde{\mathrm{x}}_{2}$ & $\tilde{\mathrm{x}}_{3}$ & $\tilde{\mathrm{x}}_{4}$ & $\mathrm{NK}$ \\
\hline $\mathrm{Z}_{\mathrm{n}}$ & $-\left(\mathrm{u}_{\mathrm{n}}\right)$ & $-\left(\mathrm{v}_{\mathrm{n}}\right)$ & $(0)$ & $(0)$ & $(0)$ \\
\hline$\tilde{\mathrm{x}}_{3}$ & $\left(\mathrm{x}_{1}, \mathrm{x}_{2}, \mathrm{x}_{3}, \mathrm{x}_{4}\right)$ & $\left(\mathrm{y}_{1}, \mathrm{y}_{2}, \mathrm{y}_{3}, \mathrm{y}_{4}\right)$ & $(1,1,1,1)$ & $(0,0,0,0)$ & $\left(\mathrm{z}_{1}, \mathrm{z}_{2}, \mathrm{z}_{3}, \mathrm{z}_{4}\right)$ \\
\hline$\tilde{\mathrm{x}}_{4}$ & $\left(\mathrm{p}_{1}, \mathrm{p}_{2}, \mathrm{p}_{3}, \mathrm{p}_{4}\right)$ & $\left(\mathrm{q}_{1}, \mathrm{q}_{2}, \mathrm{q}_{3}, \mathrm{q}_{4}\right)$ & $(0,0,0,0)$ & $(1,1,1,1)$ & $\left(\mathrm{r}_{1}, \mathrm{r}_{2}, \mathrm{r}_{3}, \mathrm{r}_{4}\right)$ \\
\hline
\end{tabular}

\section{Langkah 3}

Menentukan elemen pivot pada tabel simplex dengan menggunakan pendekatan aturan fungsi ranking, sehingga penentuan besar atau kecilnya suatu bilangan trapesium dengan bilangan trapesium lainnya pada tabel simplex lebih akurat. Menentukan variabel baris kunci (variabel yang memiliki rasio atau titik potong terkecil positif selain nol), dengan ditemukannya kolom kunci dan baris kuncinya, maka titik potong dari keduanya akan secara otomatis menjadi elemen pivot. 


\section{Muhammad Amrullah}

Variabel sebarisnya akan menjadi persamaan pivot lama, dan variabel dari baris yang lainnya akan menjadi persamaan lama.

$\tilde{\mathrm{x}}_{3}=\frac{\left(\mathrm{z}_{1}, \mathrm{z}_{2}, \mathrm{z}_{3}, \mathrm{z}_{4}\right)}{\left(\mathrm{y}_{1}, \mathrm{y}_{2}, \mathrm{y}_{3}, \mathrm{y}_{4}\right)}=\left(\frac{\mathrm{z}_{1}}{\mathrm{y}_{4}}, \frac{\mathrm{z}_{2}}{\mathrm{y}_{3}}, \frac{\mathrm{z}_{3}}{\mathrm{y}_{2}}, \frac{\mathrm{z}_{4}}{\mathrm{y}_{1}}\right)$ (memenuhi kriteria)

$\tilde{\mathrm{X}}_{4}=\frac{\left(\mathrm{r}_{1}, \mathrm{r}_{2}, \mathrm{r}_{3}, \mathrm{r}_{4}\right)}{\left(\mathrm{q}_{1}, \mathrm{q}_{2}, \mathrm{q}_{3}, \mathrm{q}_{4}\right)}=\left(\frac{\mathrm{r}_{1}}{\mathrm{q}_{4}}, \frac{\mathrm{r}_{2}}{\mathrm{q}_{3}}, \frac{\mathrm{r}_{3}}{\mathrm{q}_{2}}, \frac{\mathrm{r}_{4}}{\mathrm{q}_{1}}\right)$ (memenuhi kriteria)

$\mathrm{Z}_{\mathrm{n}}=\frac{(0,0,0,0)}{\left(-\mathrm{u}_{4},-\mathrm{u}_{3}-\mathrm{u}_{2},-\mathrm{u}_{1}\right)}=(0,0,0,0)$ (tidak memenuhi kriteria karena hasilnya 0$)$

$$
\begin{aligned}
\mathrm{R}\left(\frac{\left(\mathrm{z}_{1}, \mathrm{z}_{2}, \mathrm{z}_{3}, \mathrm{z}_{4}\right)}{\left(\mathrm{y}_{1}, \mathrm{y}_{2}, \mathrm{y}_{3}, \mathrm{y}_{4}\right)}\right) & =\mathrm{R}\left(\frac{\mathrm{z}_{1}}{\mathrm{y}_{4}}, \frac{\mathrm{z}_{2}}{\mathrm{y}_{3}}, \frac{\mathrm{z}_{3}}{\mathrm{y}_{2}}, \frac{\mathrm{z}_{4}}{\mathrm{y}_{1}}\right) \\
& =\frac{1}{2}\left(\left(\frac{\mathrm{z}_{2}}{\mathrm{y}_{3}}\right)+\left(\frac{\mathrm{z}_{3}}{\mathrm{y}_{2}}\right)\right)+\frac{1}{2}\left(\left(\left(\frac{\mathrm{z}_{4}}{\mathrm{y}_{1}}\right)+\left(\frac{\mathrm{z}_{3}}{\mathrm{y}_{2}}\right)\right)-\left(\left(\frac{\mathrm{z}_{2}}{\mathrm{y}_{3}}\right)+\left(\frac{\mathrm{z}_{1}}{\mathrm{y}_{4}}\right)\right)\right),
\end{aligned}
$$

dan

$$
\begin{aligned}
\mathrm{R}\left(\frac{\left(\mathrm{r}_{1}, \mathrm{r}_{2}, \mathrm{r}_{3}, \mathrm{r}_{4}\right)}{\left(\mathrm{q}_{1}, \mathrm{q}_{2}, \mathrm{q}_{3}, \mathrm{q}_{4}\right)}\right) & =\mathrm{R}\left(\frac{\mathrm{r}_{1}}{\mathrm{q}_{4}}, \frac{\mathrm{r}_{2}}{\mathrm{q}_{3}}, \frac{\mathrm{r}_{3}}{\mathrm{q}_{2}}, \frac{\mathrm{r}_{4}}{\mathrm{q}_{1}}\right) \\
& =\frac{1}{2}\left(\left(\frac{\mathrm{r}_{2}}{\mathrm{q}_{3}},\right)+\left(\frac{\mathrm{r}_{3}}{\mathrm{q}_{2}}\right)\right)+\frac{1}{2}\left(\left(\left(\frac{\mathrm{r}_{4}}{\mathrm{q}_{1}}\right)+\left(\frac{\mathrm{r}_{3}}{\mathrm{q}_{2}}\right)\right)-\left(\left(\frac{\mathrm{r}_{2}}{\mathrm{q}_{3}}\right)+\left(\frac{\mathrm{r}_{1}}{\mathrm{q}_{4}}\right)\right)\right) .
\end{aligned}
$$

Dari perhitungan di atas, misalkan hasil dari $\tilde{\mathrm{X}}_{4}$ memiliki rasio atau titik potong terkecil positif maka variabel sebarisnya menjadi baris kunci, dan titik potong antara baris kunci dan kolom kunci tersebut menghasilkan elemen pivot dengan koordinat baris 2 kolom 2 sesuai tabel berikut:

Tabel 4.3 Pivot 1

\begin{tabular}{|c|c|c|c|c|c|}
\hline & $\tilde{\mathrm{x}}_{1}$ & $\tilde{\mathrm{x}}_{2}$ & $\tilde{\mathrm{x}}_{3}$ & $\tilde{\mathrm{x}}_{4}$ & $\mathrm{NK}$ \\
\hline $\mathrm{Z}_{\mathrm{n}}$ & $-\left(\mathrm{u}_{\mathrm{n}}\right)$ & $-\left(\mathrm{v}_{\mathrm{n}}\right)$ & $(0)$ & $(0)$ & $(0)$ \\
\hline$\tilde{\mathrm{x}}_{3}$ & $\left.\mathrm{f}_{1}, \mathrm{x}_{2}, \mathrm{x}_{3}, \mathrm{x}_{4}\right)$ & $\left(\mathrm{y}_{1}, \mathrm{y}_{2}, \mathrm{y}_{3}, \mathrm{y}_{4}\right)$ & $(1,1,1,1)$ & $(0,0,0,0)$ & $\left(\mathrm{z}_{1}, \mathrm{z}_{2}, \mathrm{z}_{3}, \mathrm{z}_{4}\right)$ \\
\hline$\tilde{\mathrm{x}}_{4}$ & $\left(\mathrm{p}_{1}, \mathrm{p}_{2}, \mathrm{p}_{3}, \mathrm{p}_{4}\right)$ & $\left(\mathrm{q}_{1}, \mathrm{q}_{2}, \mathrm{q}_{3}, \mathrm{q}_{4}\right)$ & $(0,0,0,0)$ & $(1,1,1,1)$ & $\left(\mathrm{r}_{1}, \mathrm{r}_{2}, \mathrm{r}_{3}, \mathrm{r}_{4}\right)$ \\
\hline
\end{tabular}

\section{Langkah 4}

Menghitung persamaan pivot baru dengan persamaan lainnya.

Untuk persamaan pivot:

Persamaan pivot baru $=\frac{\text { persamaan pivot lama }}{\text { elemen pivot }}$

Untuk persamaan selain pivot:

Persamaan baru $=($ persamaan lama) $-($ koefisien kolom kunci) $\mathrm{x}$ (persamaan pivot baru).

Karena $\tilde{\mathrm{X}}_{4}$ merupakan baris kunci dan barisnya menjadi persamaan pivot lama, maka persamaan pivot barunya sebagai berikut :

$\tilde{\mathrm{x}}_{1}=\frac{\left(\mathrm{p}_{1}, \mathrm{p}_{2}, \mathrm{p}_{3}, \mathrm{p}_{4}\right)}{\left(\mathrm{q}_{1}, \mathrm{q}_{2}, \mathrm{q}_{3}, \mathrm{q}_{4}\right)}=\left(\frac{\mathrm{p}_{1}}{\mathrm{q}_{4}}, \frac{\mathrm{p}_{2}}{\mathrm{q}_{3}}, \frac{\mathrm{p}_{3}}{\mathrm{q}_{2}}, \frac{\mathrm{p}_{4}}{\mathrm{q}_{1}}\right)$

$\tilde{\mathrm{x}}_{2}=\frac{\left(\mathrm{q}_{1}, \mathrm{q}_{2}, \mathrm{q}_{3}, \mathrm{q}_{4}\right)}{\left(\mathrm{q}_{1}, \mathrm{q}_{2}, \mathrm{q}_{3}, \mathrm{q}_{4}\right)}=\left(\frac{\mathrm{q}_{1}}{\mathrm{q}_{4}}, \frac{\mathrm{q}_{2}}{\mathrm{q}_{3}}, \frac{\mathrm{q}_{3}}{\mathrm{q}_{2}}, \frac{\mathrm{q}_{4}}{\mathrm{q}_{1}}\right)$

$\tilde{\mathrm{X}}_{3}=\frac{(0,0,0,0)}{\left(\mathrm{q}_{1}, \mathrm{q}_{2}, \mathrm{q}_{3}, \mathrm{q}_{4}\right)}=(0,0,0,0)$

$\tilde{\mathrm{X}}_{4}=\frac{(1,1,1,1)}{\left(\left(\mathrm{q}_{1}, \mathrm{q}_{2}, \mathrm{q}_{3}, \mathrm{q}_{4}\right)\right)}=\left(\frac{1}{\mathrm{q}_{4}}, \frac{1}{\mathrm{q}_{3}}, \frac{1}{\mathrm{q}_{2}}, \frac{1}{\mathrm{q}_{1}}\right)$

$\mathrm{NK}=\frac{\left(\mathrm{r}_{1}, \mathrm{r}_{2}, \mathrm{r}_{3}, \mathrm{r}_{4}\right)}{\left(\mathrm{q}_{1}, \mathrm{q}_{2}, \mathrm{q}_{3}, \mathrm{q}_{4}\right)}=\left(\frac{\mathrm{r}_{1}}{\mathrm{q}_{4}}, \frac{\mathrm{r}_{2}}{\mathrm{q}_{3}}, \frac{\mathrm{r}_{3}}{\mathrm{q}_{2}}, \frac{\mathrm{r}_{4}}{\mathrm{q}_{1}}\right)$

sehingga dari perhitungan di atas, dapat ditulis pada tabel berikut :

Tabel 4.4 Persamaan baru $\tilde{\mathrm{x}}_{4}$ menjadi $\tilde{\mathrm{x}}_{2}$

\begin{tabular}{|c|c|c|c|c|c|}
\hline & $\tilde{\mathrm{x}}_{1}$ & $\tilde{\mathrm{x}}_{2}$ & $\tilde{\mathrm{x}}_{3}$ & $\tilde{\mathrm{x}}_{4}$ & $\mathrm{NK}$ \\
\hline $\mathrm{Z}_{\mathrm{n}}$ & & & & & \\
\hline$\tilde{\mathrm{x}}_{3}$ & & & & & \\
\hline$\tilde{\mathrm{x}}_{2}$ & (A.1) & (A.2) & (A.3) & (A.4) & (A.5) \\
\hline
\end{tabular}




\section{Muhammad Amrullah}

sedangkan persamaan lainnya yaitu persamaan $\begin{array}{lllll}Z_{n} & \text { dan } & \tilde{x}_{3} & \text { yang menjadi persamaan }\end{array}$ lama, persamaan barunya yaitu:

untuk persamaan $Z_{\mathrm{n}}$ di antaranya:

$$
\begin{aligned}
& \tilde{\mathrm{x}}_{1}=\left(-\mathrm{u}_{\mathrm{n}}\right)-\left(-\mathrm{v}_{\mathrm{n}}\right) \times(\text { A.1 }) \\
& =\left(-u_{n}\right)-\left(-v_{n}\right) \times\left(\frac{p_{1}}{q_{4}}, \frac{p_{2}}{q_{3}}, \frac{p_{3}}{q_{2}}, \frac{p_{4}}{q_{1}}\right) \\
& =\left(-\mathrm{u}_{\mathrm{n}}\right)-\left(-\mathrm{v}_{\mathrm{n}}\left(\frac{\mathrm{p}_{1}}{\mathrm{q}_{4}}\right),-\mathrm{v}_{\mathrm{n}}\left(\frac{\mathrm{p}_{2}}{\mathrm{q}_{3}}\right),-\mathrm{v}_{\mathrm{n}}\left(\frac{\mathrm{p}_{3}}{\mathrm{q}_{2}}\right),-\mathrm{v}_{\mathrm{n}}\left(\frac{\mathrm{p}_{4}}{\mathrm{q}_{1}}\right)\right) \\
& =\left(-\mathrm{u}_{\mathrm{n}}\right)-\mathrm{R}\left(-\mathrm{v}_{\mathrm{n}}\left(\frac{\mathrm{p}_{1}}{\mathrm{q}_{4}}\right),-\mathrm{v}_{\mathrm{n}}\left(\frac{\mathrm{p}_{2}}{\mathrm{q}_{3}}\right),-\mathrm{V}_{\mathrm{n}}\left(\frac{\mathrm{p}_{3}}{\mathrm{q}_{2}}\right),-\mathrm{v}_{\mathrm{n}}\left(\frac{\mathrm{p}_{4}}{\mathrm{q}_{1}}\right)\right) \\
& \tilde{\mathrm{x}}_{2}=\left(-\mathrm{v}_{\mathrm{n}}\right)-\left(\left(-\mathrm{v}_{\mathrm{n}}\right)\right) \times(\text { A.2) }) \\
& \left.=\left(-\mathrm{v}_{\mathrm{n}}\right)-\left(\left(-\mathrm{v}_{\mathrm{n}}\right)\right) \times\left(\left(\frac{\mathrm{q}_{1}}{\mathrm{q}_{4}}, \frac{\mathrm{q}_{2}}{\mathrm{q}_{3}}, \frac{\mathrm{q}_{3}}{\mathrm{q}_{2}}, \frac{\mathrm{q}_{4}}{\mathrm{q}_{1}}\right)\right)\right) \\
& =\left(-\mathrm{v}_{\mathrm{n}}\right)-\left(-\mathrm{v}_{\mathrm{n}}\left(\frac{\mathrm{q}_{1}}{\mathrm{q}_{4}}\right),-\mathrm{v}_{\mathrm{n}}\left(, \frac{\mathrm{q}_{2}}{\mathrm{q}_{3}}\right),-\mathrm{v}_{\mathrm{n}}\left(\frac{\mathrm{q}_{3}}{\mathrm{q}_{2}}\right),-\mathrm{v}_{\mathrm{n}}\left(\frac{\mathrm{q}_{4}}{\mathrm{q}_{1}}\right)\right) \\
& =\left(-\mathrm{v}_{\mathrm{n}}\right)-\mathrm{R}\left(-\mathrm{v}_{\mathrm{n}}\left(\frac{\mathrm{q}_{1}}{\mathrm{q}_{4}}\right),-\mathrm{v}_{\mathrm{n}}\left(\frac{\mathrm{q}_{2}}{\mathrm{q}_{3}}\right),-\mathrm{v}_{\mathrm{n}}\left(\frac{\mathrm{q}_{3}}{\mathrm{q}_{2}}\right),-\mathrm{v}_{\mathrm{n}}\left(\frac{\mathrm{q}_{4}}{\mathrm{q}_{1}}\right)\right) \\
& \tilde{\mathrm{x}}_{3}=(0)-\left(\left(-\mathrm{V}_{\mathrm{n}}\right) \times(\text { A.3 })\right) \\
& =(0)-\mathrm{R}\left(\left(-\mathrm{V}_{\mathrm{n}}\right) \times(\mathrm{A} .3)\right) \\
& \tilde{\mathrm{x}}_{4}=(0)-\left(\left(-\mathrm{v}_{4},-\mathrm{V}_{3},-\mathrm{V}_{2},-\mathrm{v}_{1}\right) \times(\text { A.4 })\right) \\
& =(0)-\left(\left(-\mathrm{v}_{4},-\mathrm{v}_{3},-\mathrm{v}_{2},-\mathrm{v}_{1}\right) \times\left(\frac{1}{\mathrm{q}_{4}}, \frac{1}{\mathrm{q}_{3}}, \frac{1}{\mathrm{q}_{2}}, \frac{1}{\mathrm{q}_{1}}\right)\right) \\
& =0-\mathrm{R}\left(\left(-\mathrm{v}_{4}\right) \frac{1}{\mathrm{q}_{4}},-\left(-\mathrm{v}_{3}\right) \frac{1}{\mathrm{q}_{3}},-\left(-\mathrm{v}_{2}\right) \frac{1}{\mathrm{q}_{2}},-\left(-\mathrm{v}_{1}\right) \frac{1}{\mathrm{q}_{1}}\right) \\
& \mathrm{NK}=(0)-\left(\left(-\mathrm{v}_{4},-\mathrm{v}_{3},-\mathrm{v}_{2},-\mathrm{v}_{1}\right) \times(\text { A.5) })\right. \\
& =(0)-\left(\left(-\mathrm{v}_{4},-\mathrm{v}_{3},-\mathrm{v}_{2},-\mathrm{v}_{1}\right) \times\left(\frac{\mathrm{r}_{1}}{\mathrm{q}_{4}}, \frac{\mathrm{r}_{2}}{\mathrm{q}_{3}}, \frac{\mathrm{r}_{3}}{\mathrm{q}_{2}}, \frac{\mathrm{r}_{4}}{\mathrm{q}_{1}}\right)\right. \\
& =(0)-\mathrm{R}\left(\left(-\mathrm{v}_{4}\right) \frac{\mathrm{r}_{1}}{\mathrm{q}_{4}},\left(-\mathrm{v}_{3}\right) \frac{\mathrm{r}_{2}}{\mathrm{q}_{3}},\left(-\mathrm{v}_{2}\right) \frac{\mathrm{r}_{3}}{\mathrm{q}_{2}},\left(-\mathrm{v}_{1}\right) \frac{\mathrm{r}_{4}}{\mathrm{q}_{1}}\right)
\end{aligned}
$$

Untuk persamaan $\tilde{\mathrm{x}}_{3}$, diantaranya:

$$
\begin{aligned}
& \tilde{\mathrm{x}}_{1} \quad=\left(\mathrm{x}_{1}, \mathrm{x}_{2}, \mathrm{x}_{3}, \mathrm{x}_{4}\right)-\left(\left(\mathrm{y}_{1}, \mathrm{y}_{2}, \mathrm{y}_{3}, \mathrm{y}_{4}\right) \mathrm{x}(\mathrm{A} .1)\right) \\
& =\left(\mathrm{x}_{1}, \mathrm{x}_{2}, \mathrm{x}_{3}, \mathrm{x}_{4}\right)-\left(\left(\mathrm{y}_{1}, \mathrm{y}_{2}, \mathrm{y}_{3}, \mathrm{y}_{4}\right) \times\left(\frac{\mathrm{p}_{1}}{\mathrm{q}_{4}}, \frac{\mathrm{p}_{2}}{\mathrm{q}_{3}}, \frac{\mathrm{p}_{3}}{\mathrm{q}_{2}}, \frac{\mathrm{p}_{4}}{\mathrm{q}_{1}}\right)\right) \\
& =\left(\mathrm{x}_{1}, \mathrm{x}_{2}, \mathrm{x}_{3}, \mathrm{x}_{4}\right)-\left(\left(\mathrm{y}_{1}\right) \frac{\mathrm{p}_{1}}{\mathrm{q}_{4}},\left(\mathrm{y}_{2}\right) \frac{\mathrm{p}_{2}}{\mathrm{q}_{3}},\left(\mathrm{y}_{3}\right) \frac{\mathrm{p}_{3}}{\mathrm{q}_{2}},\left(\mathrm{y}_{4}\right) \frac{\mathrm{p}_{4}}{\mathrm{q}_{1}}\right) \\
& =\left(x_{1}-\left(y_{4}\right) \frac{p_{4}}{q_{1}}, x_{2}-\left(y_{3}\right) \frac{p_{3}}{q_{2}}, x_{3}-\left(y_{2}\right) \frac{p_{2}}{q_{3}}, x_{4}-\left(y_{1}\right) \frac{p_{1}}{q_{4}}\right) \\
& \tilde{\mathrm{x}}_{2} \quad=\left(\mathrm{y}_{1}, \mathrm{y}_{2}, \mathrm{y}_{3}, \mathrm{y}_{4}\right)-\left(\left(\mathrm{y}_{1}, \mathrm{y}_{2}, \mathrm{y}_{3}, \mathrm{y}_{4}\right) \mathrm{x}\right. \text { (A.2)) } \\
& =\left(y_{1}, y_{2}, y_{3}, y_{4}\right)-\left(\left(y_{1}, y_{2}, y_{3}, y_{4}\right) \times\left(\frac{q_{1}}{q_{4}}, \frac{q_{2}}{q_{3}}, \frac{q_{3}}{q_{2}}, \frac{q_{4}}{q_{1}}\right)\right) \\
& =\left(\mathrm{y}_{1}, \mathrm{y}_{2}, \mathrm{y}_{3}, \mathrm{y}_{4}\right)-\left(\mathrm{y}_{1}\left(\frac{\mathrm{q}_{1}}{\mathrm{q}_{4}}\right), \mathrm{y}_{2}\left(\frac{\mathrm{q}_{2}}{\mathrm{q}_{3}}\right), \mathrm{y}_{3}\left(\frac{\mathrm{q}_{3}}{\mathrm{q}_{2}}\right), \mathrm{y}_{4}\left(\frac{\mathrm{q}_{4}}{\mathrm{q}_{1}}\right)\right) \\
& \tilde{\mathrm{x}}_{3} \quad=(1,1,1,1)-\left(\left(\mathrm{y}_{1}, \mathrm{y}_{2}, \mathrm{y}_{3}, \mathrm{y}_{4}\right) \times(\text { A.3 })\right) \\
& =(1,1,1,1)-\left(\left(\mathrm{y}_{1}, \mathrm{y}_{2}, \mathrm{y}_{3}, \mathrm{y}_{4}\right) \times(0,0,0,0)\right. \\
& =(1,1,1,1) \\
& \tilde{\mathrm{x}}_{4} \quad=(0,0,0,0)-\left(\left(\mathrm{y}_{1}, \mathrm{y}_{2}, \mathrm{y}_{3}, \mathrm{y}_{4}\right) \times(\mathrm{A} .4)\right) \\
& =(0,0,0,0)-\left(\left(\mathrm{y}_{1}, \mathrm{y}_{2}, \mathrm{y}_{3}, \mathrm{y}_{4}\right) \times\left(\frac{1}{\mathrm{q}_{4}}, \frac{1}{\mathrm{q}_{3}}, \frac{1}{\mathrm{q}_{2}}, \frac{1}{\mathrm{q}_{1}}\right)\right) \\
& =(0,0,0,0)-\left(\mathrm{y}_{1}\left(\frac{1}{\mathrm{q}_{4}}\right), \mathrm{y}_{2}\left(\frac{1}{\mathrm{q}_{3}}\right), \mathrm{y}_{3}\left(\frac{1}{\mathrm{q}_{2}}\right), \mathrm{y}_{4}\left(\frac{1}{\mathrm{q}_{1}}\right)\right) \\
& =\left(-\mathrm{y}_{4}\left(\frac{1}{\mathrm{q}_{1}}\right),-\mathrm{y}_{3}\left(\frac{1}{\mathrm{q}_{2}}\right),-\mathrm{y}_{2}\left(\frac{1}{\mathrm{q}_{3}}\right),-\left(\mathrm{y}_{1}\left(\frac{1}{\mathrm{q}_{4}}\right)\right)\right. \\
& \mathrm{NK}=\left(\mathrm{z}_{1}, \mathrm{z}_{2}, \mathrm{z}_{3}, \mathrm{z}_{4}\right)-\left(\left(\mathrm{y}_{1}, \mathrm{y}_{2}, \mathrm{y}_{3}, \mathrm{y}_{4}\right) \times(\mathrm{A} .5)\right) \\
& =\left(z_{1}, z_{2}, z_{3}, z_{4}\right)-\left(\left(y_{1}, y_{2}, y_{3}, y_{4}\right) x\left(\frac{r_{1}}{q_{4}}, \frac{r_{2}}{q_{3}}, \frac{r_{3}}{q_{2}}, \frac{r_{4}}{q_{1}}\right)\right) \\
& =\left(\mathrm{z}_{1}, \mathrm{z}_{2}, \mathrm{z}_{3}, \mathrm{z}_{4}\right)-\left(\mathrm{y}_{1}\left(\frac{\mathrm{r}_{1}}{\mathrm{q}_{4}}\right), \mathrm{y}_{2}\left(\frac{\mathrm{r}_{2}}{\mathrm{q}_{3}}\right), \mathrm{y}_{3}\left(\frac{\mathrm{r}_{3}}{\mathrm{q}_{2}}\right), \mathrm{y}_{4}\left(\frac{\mathrm{r}_{4}}{\mathrm{q}_{1}}\right)\right) \\
& \left.=\left(\mathrm{z}_{1}-\mathrm{y}_{4}\left(\frac{\mathrm{r}_{4}}{\mathrm{q}_{1}}\right), \mathrm{z}_{2}-\mathrm{y}_{3}\left(\frac{\mathrm{r}_{3}}{\mathrm{q}_{2}}\right), \mathrm{z}_{3}-\mathrm{y}_{2}\left(\frac{\mathrm{r}_{2}}{\mathrm{q}_{3}}\right), \mathrm{z}_{4}-\mathrm{y}_{1}\left(\frac{\mathrm{r}_{1}}{\mathrm{q}_{4}}\right)\right)\right)
\end{aligned}
$$


sehingga dari perhitungan di atas, hasil iterasi pertama dapat ditulis sesuai tabel berikut:

Tabel 4.5 Persamaan baru $Z_{\mathrm{n}}$ dan $\tilde{\mathrm{x}}_{3}$

\begin{tabular}{|c|c|c|c|c|c|}
\hline & $\tilde{\mathrm{x}}_{1}$ & $\tilde{\mathrm{x}}_{2}$ & $\tilde{\mathrm{x}}_{3}$ & $\tilde{\mathrm{x}}_{4}$ & $\mathrm{NK}$ \\
\hline $\mathrm{Z}_{\mathrm{n}}$ & (B.1) & (B.2) & (B.3) & (B.4) & (B.5) \\
\hline$\tilde{\mathrm{x}}_{3}$ & (C.1) & (C.2) & (C.3) & (C.4) & (C.5) \\
\hline$\tilde{\mathrm{x}}_{2}$ & (A.1) & (A.2) & (A.3) & (A.4) & (A.5) \\
\hline
\end{tabular}

karena pada operasi bilangan kabur trapesium hasil perhitungan dari operasinya semakin melebar yaitu yang positif semakin positif dan yang negatif semakin negatif jika iterasi diteruskan, maka selanjutnya dilakukan defuzyfikasi untuk setiap elemen dalam tabel simplex untuk bisa dilanjutkan iterasi berikutnya. Pada iterasi pertama diperoleh solusi untuk $\tilde{\mathrm{x}}_{2}$ dan $\mathrm{Z}_{\mathrm{n}}$ yaitu:

$$
\begin{aligned}
\tilde{\mathrm{x}}_{2} & =\mathrm{A} .5 \\
& =\left(\frac{\mathrm{r}_{1}}{\mathrm{q}_{4}}, \frac{\mathrm{r}_{2}}{\mathrm{q}_{3}}, \frac{\mathrm{r}_{3}}{\mathrm{q}_{2}}, \frac{\mathrm{r}_{4}}{\mathrm{q}_{1}}\right) \\
\mathrm{Z}_{\mathrm{n}} & =(\mathrm{B} .5) \\
& =(0)-\mathrm{R}\left(\left(-\mathrm{v}_{4}\right) \frac{\mathrm{r}_{1}}{\mathrm{q}_{4}},\left(-\mathrm{v}_{3}\right) \frac{\mathrm{r}_{2}}{\mathrm{q}_{3}},\left(-\mathrm{v}_{2}\right) \frac{\mathrm{r}_{3}}{\mathrm{q}_{2}},\left(-\mathrm{v}_{1}\right) \frac{\mathrm{r}_{4}}{\mathrm{q}_{1}}\right) .
\end{aligned}
$$

Hasil ini berlaku untuk kasus yang elemen pivotnya terletak pada perpotongan antara kolom $\tilde{\mathrm{x}}_{2}$ dengan baris $\tilde{\mathrm{x}}_{4}$ (Tabel 4.3).

Untuk kasus lain dengan cara yang sama jika elemen pivotnya terletak pada perpotongan antara kolom $\tilde{\mathrm{x}}_{2}$ dengan baris $\tilde{\mathrm{x}}_{3}$ (Tabel 4.6), maka niali maksimum untuk $\tilde{\mathrm{x}}_{2}$ dan $\mathrm{Z}_{\mathrm{n}}$ sesuai tabel berikut:

Tabel 4.6 pivot 2

\begin{tabular}{|c|c|c|c|c|c|}
\hline & $\tilde{\mathrm{x}}_{1}$ & $\tilde{\mathrm{x}}_{2}$ & $\tilde{\mathrm{x}}_{3}$ & $\tilde{\mathrm{x}}_{4}$ & $\mathrm{NK}$ \\
\hline $\mathrm{Z}_{\mathrm{n}}$ & $\left(-\mathrm{u}_{\mathrm{n}}\right)$ & $\left(-\mathrm{v}_{\mathrm{n}}\right)$ & $(0)$ & $(0)$ & $(0)$ \\
\hline$\tilde{\mathrm{x}}_{3}$ & $\left(\mathrm{x}_{1}, \mathrm{x}_{2}, \mathrm{x}_{3}, \mathrm{x}_{4}\right)$ & $\left(\mathrm{y}_{1}, \mathrm{y}_{2}, \mathrm{y}_{3}, \mathrm{y}_{4}\right)$ & $(1,1,1,1)$ & $(0,0,0,0)$ & $\left(\mathrm{z}_{1}, \mathrm{z}_{2}, \mathrm{z}_{3}, \mathrm{z}_{4}\right)$ \\
\hline$\tilde{\mathrm{x}}_{4}$ & $\left(\mathrm{p}_{1}, \mathrm{p}_{2}, \mathrm{p}_{3}, \mathrm{p}_{4}\right)$ & $\left(\mathrm{q}_{1}, \mathrm{q}_{2}, \mathrm{q}_{3}, \mathrm{q}_{4}\right)$ & $(0,0,0,0)$ & $(1,1,1,1)$ & $\left(\mathrm{r}_{1}, \mathrm{r}_{2}, \mathrm{r}_{3}, \mathrm{r}_{4}\right)$ \\
\hline
\end{tabular}

$$
\begin{aligned}
\tilde{\mathrm{x}}_{2} & =\left(\frac{\mathrm{z}_{1}}{\mathrm{y}_{4}}, \frac{\mathrm{z}_{2}}{\mathrm{y}_{3}}, \frac{\mathrm{z}_{3}}{\mathrm{y}_{2}}, \frac{\mathrm{z}_{4}}{\mathrm{y}_{1}}\right) \\
\mathrm{Z}_{\mathrm{n}} & =(0)-\left(\left(-\mathrm{v}_{\mathrm{n}}\right) \mathrm{x}\left(\frac{\mathrm{z}_{1}}{\mathrm{y}_{4}}, \frac{\mathrm{z}_{2}}{\mathrm{y}_{3}}, \frac{\mathrm{z}_{3}}{\mathrm{y}_{2}}, \frac{\mathrm{z}_{4}}{\mathrm{y}_{1}}\right)\right. \\
& =(0)-\mathrm{R}\left(\left(-\mathrm{v}_{4}\right) \frac{\mathrm{z}_{1}}{\mathrm{y}_{4}},\left(-\mathrm{v}_{3}\right) \frac{\mathrm{z}_{2}}{\mathrm{y}_{3}},\left(-\mathrm{v}_{2}\right) \frac{\mathrm{z}_{3}}{\mathrm{y}_{2}},\left(-\mathrm{v}_{1}\right), \frac{\mathrm{z}_{4}}{\mathrm{y}_{1}}\right) .
\end{aligned}
$$

Untuk kasus lain dengan cara yang sama jika elemen pivotnya terletak pada perpotongan antara kolom $\tilde{\mathrm{x}}_{1}$ dengan baris $\tilde{\mathrm{x}}_{3}$ (Tabel 4.7), maka nilai maksimum untuk $\tilde{\mathrm{x}}_{1}$ dan $Z_{\mathrm{n}}$ yaitu:

Tabel 4.7 Pivot 3

\begin{tabular}{|l|c|c|c|c|c|}
\hline & $\tilde{\mathrm{x}}_{1}$ & $\tilde{\mathrm{x}}_{2}$ & $\tilde{\mathrm{x}}_{3}$ & $\tilde{\mathrm{x}}_{4}$ & $\mathrm{NK}$ \\
\hline $\mathrm{Z}_{\mathrm{n}}$ & $\left(-\mathrm{u}_{\mathrm{n}}\right)$ & $\left(-\mathrm{v}_{\mathrm{n}}\right)$ & $(0)$ & $(0)$ & $(0)$ \\
\hline$\tilde{\mathrm{x}}_{3}$ & $\left(\mathrm{x}_{1}, \mathrm{x}_{2}, \mathrm{x}_{3}, \mathrm{x}_{4}\right)$ & $\left(\mathrm{y}_{1}, \mathrm{y}_{2}, \mathrm{y}_{3}, \mathrm{y}_{4}\right)$ & $(1,1,1,1)$ & $(0,0,0,0)$ & $\left(\mathrm{z}_{1}, \mathrm{z}_{2}, \mathrm{z}_{3}, \mathrm{z}_{4}\right)$ \\
\hline$\tilde{\mathrm{x}}_{4}$ & $\left(\mathrm{p}_{1}, \mathrm{p}_{2}, \mathrm{p}_{3}, \mathrm{p}_{4}\right)$ & $\left(\mathrm{q}_{1}, \mathrm{q}_{2}, \mathrm{q}_{3}, \mathrm{q}_{4}\right)$ & $(0,0,0,0)$ & $(1,1,1,1)$ & $\left(\mathrm{r}_{1}, \mathrm{r}_{2}, \mathrm{r}_{3}, \mathrm{r}_{4}\right)$ \\
\hline
\end{tabular}

$$
\begin{aligned}
& \tilde{\mathrm{x}}_{1} \quad=\frac{\mathrm{z}_{1}}{\mathrm{x}_{4}}, \frac{\mathrm{z}_{2}}{\mathrm{x}_{3}}, \frac{\mathrm{z}_{3}}{\mathrm{x}_{2}}, \frac{\mathrm{z}_{4}}{\mathrm{x}_{1}} \\
& \mathrm{Z}_{\mathrm{n}}=(0)-\left(\left(-\mathrm{u}_{4},-\mathrm{u}_{3},-\mathrm{u}_{2},-\mathrm{u}_{1}\right) \mathrm{x}\left(\frac{\mathrm{z}_{1}}{\mathrm{x}_{4}}, \frac{\mathrm{z}_{2}}{\mathrm{x}_{3}}, \frac{\mathrm{z}_{3}}{\mathrm{x}_{2}}, \frac{\mathrm{z}_{4}}{\mathrm{x}_{1}}\right)\right.
\end{aligned}
$$




$$
=(0)-\mathrm{R}\left(\left(-\mathrm{u}_{4}\right) \frac{\mathrm{z}_{1}}{\mathrm{x}_{4}},\left(-\mathrm{u}_{3}\right) \frac{\mathrm{z}_{2}}{\mathrm{x}_{3}},\left(-\mathrm{u}_{2}\right) \frac{\mathrm{z}_{3}}{\mathrm{x}_{2}},\left(-\mathrm{u}_{1}\right) \frac{\mathrm{z}_{4}}{\mathrm{x}_{1}}\right) .
$$

Untuk kasus lain dengan cara yang sama jika elemen pivotnya terletak pada perpotongan antara kolom $\tilde{\mathrm{X}}_{1}$ dengan baris $\tilde{\mathrm{X}}_{4}$ (Tabel 4.8), maka nilai maksimum untuk $\tilde{\mathrm{X}}_{1}$ dan $\mathrm{Z}_{\mathrm{n}}$ yaitu:

Tabel 4.8 Pivot 4

\begin{tabular}{|c|c|c|c|c|c|}
\hline & $\tilde{\mathrm{x}}_{1}$ & $\tilde{\mathrm{x}}_{3}$ & $\tilde{\mathrm{x}}_{3}$ & $\tilde{\mathrm{x}}_{4}$ & $\mathrm{NK}$ \\
\hline $\mathrm{Z}_{\mathrm{n}}$ & $\left(-\mathrm{u}_{\mathrm{n}}\right)$ & $\left(-\mathrm{v}_{\mathrm{n}}\right)$ & $(0)$ & $(0)$ & $(0)$ \\
\hline$\tilde{\mathrm{x}}_{3}$ & $\left(\mathrm{x}_{1}, \mathrm{x}_{2}, \mathrm{x}_{3}, \mathrm{x}_{4}\right)$ & $\left(\mathrm{y}_{1}, \mathrm{y}_{2}, \mathrm{y}_{3}, \mathrm{y}_{4}\right)$ & $(1,1,1,1)$ & $(0,0,0,0)$ & $\left(\mathrm{z}_{1}, \mathrm{z}_{2}, \mathrm{z}_{3}, \mathrm{z}_{4}\right)$ \\
\hline$\tilde{\mathrm{x}}_{4}$ & $\left(\mathrm{p}_{1}, \mathrm{p}_{2}, \mathrm{p}_{3}, \mathrm{p}_{4}\right)$ & $\left(\mathrm{q}_{1}, \mathrm{q}_{2}, \mathrm{q}_{3}, \mathrm{q}_{4}\right)$ & $(0,0,0,0)$ & $(1,1,1,1)$ & $\left(\mathrm{r}_{1}, \mathrm{r}_{2}, \mathrm{r}_{3}, \mathrm{r}_{4}\right)$ \\
\hline
\end{tabular}

$$
\begin{aligned}
\tilde{\mathrm{x}}_{1} & =\left(\frac{\mathrm{r}_{1}}{\mathrm{p}_{4}}, \frac{\mathrm{r}_{2}}{\mathrm{p}_{3}}, \frac{\mathrm{r}_{3}}{\mathrm{p}_{2}}, \frac{\mathrm{r}_{4}}{\mathrm{p}_{1}}\right) \\
\widetilde{\mathrm{Z}} & =(0)-\left(\left(-\mathrm{u}_{4},-\mathrm{u}_{3},-\mathrm{u}_{2},-\mathrm{u}_{1}\right) \times\left(\frac{\mathrm{r}_{1}}{\mathrm{p}_{4}}, \frac{\mathrm{r}_{2}}{\mathrm{p}_{3}}, \frac{\mathrm{r}_{3}}{\mathrm{p}_{2}}, \frac{\mathrm{r}_{4}}{\mathrm{p}_{1}}\right)\right. \\
& =(0)-\mathrm{R}\left(\left(-\mathrm{u}_{4}\right) \frac{\mathrm{r}_{1}}{\mathrm{p}_{4}},\left(-\mathrm{u}_{3}\right) \frac{\mathrm{r}_{2}}{\mathrm{p}_{3}},\left(-\mathrm{u}_{2}\right) \frac{\mathrm{r}_{3}}{\mathrm{p}_{2}},\left(-\mathrm{u}_{1}\right) \frac{\mathrm{r}_{4}}{\mathrm{p}_{1}}\right) .
\end{aligned}
$$

\section{Langkah 5}

Langkah selanjutnya adalah Proses defuzyfikasi semua elemen pada tabel simplex pada iterasi pertama (Tabel 4.5). Jika solusi optimum telah ditemukan pada iterasi pertama maka iterasi dihentikan, tapi jika belum memenuhi, maka selanjutnya dilakukan iterasi kedua sesuai tabel berikut :

Tabel 4.9 Tabel awal setelah defuzyfikasi

\begin{tabular}{|c|c|c|c|c|c|}
\hline & $\tilde{\mathrm{x}}_{1}$ & $\tilde{\mathrm{x}}_{2}$ & $\tilde{\mathrm{x}}_{3}$ & $\tilde{\mathrm{x}}_{4}$ & NK \\
\hline$\widetilde{\mathrm{Z}}$ & $\mathrm{R}(\mathrm{B} .1)$ & $\mathrm{R}(\mathrm{B} .2)$ & $\mathrm{R}(\mathrm{B} .3)$ & $\mathrm{R}(\mathrm{B} .4)$ & $\mathrm{R}(\mathrm{B} .5)$ \\
\hline$\tilde{\mathrm{x}}_{3}$ & $\mathrm{R}(\mathrm{C} .1)$ & $\mathrm{R}(\mathrm{C} .2)$ & $\mathrm{R}(\mathrm{C} .3)$ & $\mathrm{R}(\mathrm{C} .4)$ & $\mathrm{R}(\mathrm{C} .5)$ \\
\hline$\tilde{\mathrm{x}}_{2}$ & $\mathrm{R}(\mathrm{A} .1)$ & $\mathrm{R}(\mathrm{A} .2)$ & $\mathrm{R}(\mathrm{A} .3)$ & $\mathrm{R}(\mathrm{A} .4)$ & $\mathrm{R}(\mathrm{A} .5)$ \\
\hline
\end{tabular}

Setelah dilakukan tahapan-tahapan diatas, akan didapatkan solusi optimum $\widetilde{Z}=\left(Z_{1}, Z_{2}, Z_{3}\right.$, $\mathrm{Z}_{4}$ ) dari variabel $\tilde{\mathrm{x}}_{1}, \tilde{\mathrm{x}}_{2}$.

\section{Kesimpulan}

1. Memodelkan suatu masalah optimasi dengan keseluruhan unsurnya bernilai kabur dapat diselesaikan menggunakan pemrograman linier dengan metode simplex, dengan terlebih dahulu mengidentifikasi apakah masalah tersebut dapat dimodelkan kedalam model umum pemrogaraman linier kabur.

2. Pemrograman linier dengan koefisien fungsi objektif, fungsi kendala dan variabel keputusan berbentuk bilangan kabur dapat diselesaikan dengan menggunakan metode simpleks pada iterasi pertama dengan metode langsung tanpa terlebih dahulu mengubahnya menjadi pemrograman linier tegas.

3. Pemrograman linier dengan koefisien fungsi objektif, fungsi kendala dan variabel keputusan berbentuk bilangan kabur dapat diselesaikan dengan mengaplikasikan fungsi ranking : $\mathrm{R}(\widetilde{\mathrm{A}})=\frac{1}{2}\left(\mathrm{~m}_{1}+\mathrm{n}_{1}+\frac{1}{2}\left(\beta_{1}-\alpha_{1}\right)\right)$ untuk $\widetilde{\mathrm{A}}=\left(\mathrm{m}_{1}, \mathrm{n}_{1}, \alpha_{1}, \beta_{1}\right)$ dimana $\alpha_{1}=$ $\beta_{1}$ atau $\alpha_{1} \neq \beta_{1}$

\section{Daftar Pustaka}

[1] Asikin, M.,2003. Mencermati berbagai jenis permasalahan dalam program linear kabur, Jurnal matematika dan komputer, 2, 86-96.

[2] Allahviranloo, T., 2009. Solving a fully fuzzy linear programming using lexicography method and fuzzy approximate solution, Appl. Math. Model. 33.

[3] Ebrahimnejad, A., Nasseri, S. H., Lotfi F. H., dan Soltanifar, M., 2010. A primaldual method for linear programming problems with fuzzy variables, Eur. J. Ind. Eng. 4, 189-209.

[4] Hosseinzadeh, A., dan Edalatpanah, S. A., 2016. A new approach for solving fully 
fuzzy linear programming by using the lexicography method. Journal of fuzzy linear programming.

[5] Kumar, A., Kaur, J., Singh, P., 2010. Fuzzy optimal solution of fully fuzzy linear programming problems with inequality constraints, International journal of applied mathematics and computer sciences 6, 37-41.

[6] Kumar, A., dan Kaur, J., 2011. A new method for solving fuzzy linear programs with tapezoidal fuzzy numbers, Journal of fuzzy linear programming, Vol.102.

[7] Kusumadewi., 2010. Aplikasi logika kabur untuk pendukung keputusan. Yogyakarta: Graha Ilmu.

[8] Maleki, H. R., 2002. Ranking functions and their applications to fuzzy linear programming, Journal of Fuzzy Linear Programming, Far East J. Appl. Math. 4, 283301.

[9] Nasseri, S.H., Ardil, E., Yazdani, A., dan Zaefarian, R., 2007. Simplex method for solving linear programming problems with fuzzy numbers, Journal of fuzzy linear programming, Vol:12, No.10, 2007.

[10] Pandian, P., dan Jayalakshmi, M., 2010. A new for solving integer linear programming problems with fuzzy variabels, Applied mathematical sciences, vol : 4, No. 20, 997-1004.

[11] Salim, R.dan Molani, M.,2014. Representation of trapezoidal fuzzy numbers with shape function, Annals of fuzzy mathematics and informatics, volume 8, No. 1.

[12] Stanojevic, B. dan Stanojevic, M., 2009. Penalty method for fuzzy linear programming with trapezoidal numbers, Journal of operations research, 1, 149-156.

[13] Wang, L.X., 1997. A course in fuzzy systems and control, prentice-hall Int., London.

[14] Winston, W.L., 2003. Operations research: applications and algorithms, edisi-4, International Thomson Publishing, Belmont, California.

[15] Zadeh, L.A.,1965. Fuzzy Set. Information and control, 8: 338-353. California.

[16] Zimmermann, H.J., 1978. Fuzzy programming and linear programming with several objective functions, Fuzzy Sets and Systems. 